\title{
LXXXVI. Note on the analysis of energy distribution for natural radiation
}

\section{S. Lees}

To cite this article: S. Lees (1914) LXXXVI. Note on the analysis of energy distribution for natural radiation, Philosophical Magazine Series 6, 28:168, 794-798, DOI: 10.1080/14786441208635266

To link to this article: http://dx.doi.org/10.1080/14786441208635266

曲 Published online: 08 Apr 2009.

Submit your article to this journal $₫$

Џ Article views: 3

Q View related articles $₫$ 
of the secondary radiation from the two edges of the slit had to be given up, exposures with slits of various width showing the lines at the same distance. The only possibility left was that the cathode rays were not focussed in only one point of the target. Consequently, the bulb was placed in such a position that the X-rays used were emitted at a minimal angle with the surface of the target, and now both the direct and the reflected lines were simple. Possibly former cases of double lines may be referred to the same cause.

Physical Laboratory,

University of Lund, Sept. 1914.

LXXXVI. Note on the Analysis of Energy Distribution for Natural Radiation. By S. Lses, Reader in Applied Thermodynamics in the Manchester School of Technology and in the University of Manchester *.

$\S 1.7 \mathrm{THE}$ following remarks are suggested by a paper of 1 Lord Rayleigh's (Phil. Mag. xxvii. p. 466, 1889). Indeed, the theorem deduced in $\$ 4$ may be regarded as analogous to a theorem given by Rayleigh in his paper.

$\S 2$. At any point $(x, y, z)$ in a medium traversed by natural radiation corresponding to some definite temperature, we may denote the component electric forces at time $t$ by $(\mathrm{X}, \mathrm{Y}, \mathrm{Z})$. We shall consider any time-interval defined by $0<t<\mathrm{T}$, and suppose that

$$
\begin{array}{ll}
\mathrm{X}=0 \quad \text { from } t=-\infty & \text { to } t=0, \\
\mathrm{X}=\mathrm{X}(t), \quad t=0 & , t=\mathrm{T}, \\
\mathrm{X}=0 \quad, t=\mathrm{T} & , t=\infty .
\end{array}
$$

Then by Fourier's theorem,

$$
\mathrm{X}(t)=\frac{1}{\pi} \int_{p=0}^{p=\infty} \int_{u=0}^{u=\mathbf{T}} \mathrm{X}(u) \cos p(u-t) d p d u,
$$

where we assume that $\mathrm{X}$ is finite, and only has a finite number of discontinuities in the interval $0<t<T$. We may thus express $\mathrm{X}$ in the form

$$
\mathrm{X}=\int_{0}^{\infty} f(p) \cos (p t+\beta) d p, \quad . \quad .
$$

where, of course, $\beta$ may be a function of $p$, and the analysis is purely formal.

* Communicated by the Author. 
§3. Expression (ii.) may be regarded as the resultant of two trains of waves of infinite extent, travelling in opposite directions (parallel to $\mathrm{O} y$ say) in the medium, with a velocity $C$; which velocity is that of radiation of frequency $p$, if the medium is dispersive. If $y=0$ specifies the position of the place where we are studying the value of $X$, we may conveniently represent $\mathrm{X}$ by

$$
\begin{aligned}
\mathrm{X}_{y=0}=\int_{0}^{\infty} \chi_{1}(p) \cos \left[p(t+y / \mathrm{C})+\omega_{1}\right] d p \\
\quad+\int_{y=0}^{\infty} \chi_{2}(p) \cos \left[p(t-y / \mathrm{C})+\omega_{2}\right] \underset{y=0}{d p .} \text { (iii.) }
\end{aligned}
$$

The first integral may be considered as representing a train of waves stretching from $y=0$ to $y=\infty$ originally, and travelling in the negative direction $\vec{y} \overrightarrow{0}$. The second integral represents a train of waves stretching originally from $y=-\infty$ to $y=0$, and travelling in the opposito direction. Wo obviously get by comparing (ii.) and (iii.),

$$
\begin{aligned}
& \chi_{1}(p) \cos \omega_{1}+\chi_{2}(p) \cos \omega_{2}=f(p) \cos \beta, \quad . \quad \text { (iv.) } \\
& \chi_{1}(p) \sin \omega_{1}+\chi_{2}(p) \sin \omega_{2}=f^{\prime}(p) \sin \beta . . \quad \text { (v.) }
\end{aligned}
$$

We may take $\omega_{1}$ and $\omega_{2}$ as being arbitrarily hxed, when these two equations will give $\chi_{1}(p)$ and $\chi_{2}(p)$.

$\S 4$. We have now to consider the energy in the waves represented by the expressions in (iii.). The energy per unit volume due to $\mathrm{X}$ is $\epsilon \mathrm{X}^{2} / 8 \pi *$. If we consider unit area at $y=0$, normal to $\mathrm{O} y$, the amount of energy contained in the waves given by (ii.) will be that corresponding to a volume CT. We shall consider the energy $\mathrm{E}_{x}{ }^{\prime}$ corresponding to the first integral (which we shall call $\mathrm{X}_{1}$ ) in (iii.). This will be

where

$$
\mathrm{E}_{x}^{\prime}=\frac{\epsilon}{8 \pi} \int_{0}^{\infty} \mathrm{X}_{1}^{2} d y
$$

$$
\mathbf{X}_{\mathbf{I}}=\int_{0}^{\infty} \chi_{1}(p) \cos \left[p(t+y /())+\omega_{1}\right] d p .
$$

To evaluate (vi.) we re-write (vii.) in the form

$$
\mathrm{X}_{1}=\int_{0}^{\infty} \chi_{1}(q) \cos \left[q(t+y / \mathrm{C})+\omega_{1}\right] d q . \quad \text {. (viii.) }
$$

* $\epsilon$ represents the dielectric constant of the medium for frequency $p$. 
Thus $\mathrm{X}_{1}{ }^{2}$ is the product of expressions (vii.) and (viii.), whilst (vi.) becomes

$$
\begin{aligned}
\mathrm{E}_{x}{ }^{\prime}= & \frac{\epsilon}{8 \pi} \int_{y=0}^{y=\infty} \int_{p=0}^{p=\infty} \int_{q=0}^{q=\infty} \chi_{\mathrm{I}}(p) \chi_{1}(q) \cos \left[p(t+y / \mathrm{C})+\omega_{1}\right] \\
= & \frac{\epsilon}{8 \pi} \int_{0}^{\infty} \int_{0}^{\infty} \int_{0}^{\infty} \chi_{1}(p) \chi_{\mathrm{I}}(q)\{\cos [(p-q) y / \mathrm{C}] \\
& \left.\quad+\cos \left[(p+q)(t+y / \mathrm{C})+2 \omega_{1}\right]\right\} d y d p d q . \quad \text { (x.) }
\end{aligned}
$$

If we average this over a sufficiently large time, the time term in this expression can be neglected. We may now regard $\mathrm{E}_{x}{ }^{\prime}$ as the limit when $y \rightarrow \infty$, of

$$
\frac{\epsilon}{16 \pi} \int_{0}^{y} \int_{0}^{\infty} \int_{0}^{\infty} \chi_{1}(p) \chi_{1}(q) \cos (p-q) y / \mathrm{C} \cdot d y d p d q .(\mathrm{x} . a)
$$

Integrating first with respect to $y$ we obtain

$$
\mathrm{E}_{x}^{\prime}=\mathrm{Lt}_{y \rightarrow \infty} \frac{\epsilon}{16 \pi} \int_{0}^{\infty} \int_{0}^{\infty} \chi_{1}\left(p ; \chi_{1}(q) \frac{\sin [(p-q) y / \mathrm{C}]}{(p-q) / \mathrm{C}^{-}} d p d q .\right.
$$

We shall introduce a new variable $z=(p-q) y / \mathrm{C}$, so that

We then have

$$
p=q+z \mathrm{C} / y \text {. }
$$

$\mathrm{E}_{x}^{\prime}=\operatorname{Lt}_{y \rightarrow \infty} \frac{\mathrm{C} \epsilon}{16 \pi} \int_{0}^{\infty} \int_{0}^{\infty} \chi_{1}(p) \chi_{1}(q) \frac{\sin [p-q) y / \mathrm{C}]}{(p-q) y / \mathrm{C}^{-}} d[(p-q) y / \mathrm{C}] d q$,

where the $q$ integration is left second. The expression reduces to

$$
\mathrm{E}_{x}{ }^{\prime}=\frac{\mathrm{C}^{\prime} \epsilon}{16 \pi} \int_{0}^{\infty} d q \int_{z=-q y / c}^{z=\infty} \chi_{1}(q) \chi_{1}(q+z \mathrm{C} / y) \frac{\sin z}{z} d z, .
$$

where $y \rightarrow \infty$. Since, however,

$$
\int_{-\infty}^{\infty} \frac{\sin z}{z} \cdot d z=\pi
$$

we therefore get

$$
\mathrm{E}_{x}{ }^{\prime}=\frac{\mathrm{C} \epsilon}{16} \int_{0}^{\infty}\left[\chi_{1}(q)\right]^{2} \cdot d q=\frac{\mathrm{C} \epsilon}{16} \int_{0}^{\infty}\left[\chi_{1}(p)\right]^{2} \cdot d p ;
$$

provided that $\chi_{1}(q)$ is always finite, with a finite number of discontinuities. A similar expression will obviously hold for the energy due to the second integral in (iii.). Hence 
the total energy represented by (iii.) will be

$$
\underset{16}{\mathrm{C} E} \int_{0}^{\infty}\left\{\left[\chi_{1}(p)\right]^{2}+\left[\chi_{2}(p)\right]^{2}\right\} d p . \quad \text {. . (xiii.) }
$$

From (iv.) and (v.), by squaring and adding, we obtain

$$
\left[\chi_{1}(p)\right]^{2}+\left[\chi_{2}(p)\right]^{2}+2 \chi_{1}(p) \chi_{2}(p) \cos \left(\omega_{1}-\omega_{2}\right)=\left[f^{\prime}(p)\right]^{2} .
$$

We may take $\omega_{1}$ and $\omega_{2}$ as baving all values between 0 and $2 \pi$ as being equally probable. Hence averaging (xiv.), we may take

$$
\left[\chi_{1}(p)\right]^{2}+\left[\chi_{2}(p)\right]^{2}=[f(p)]^{2}
$$

Thus (xiii.) becomes

$$
\frac{\mathrm{C} \epsilon}{16} \int_{0}^{\infty}\left[f^{\prime}(p)\right]^{2} d p
$$

We may also take it that theiaverage magnetic energy in the waves is equal to the average electrical energy. Thus the total energy due to the waves represented by (ii.) is

$$
\frac{C_{\epsilon}}{8} \int_{0}^{\infty}[f(p)]^{2} \cdot d p . \quad . \quad \cdot . \quad(\mathrm{xv} .)
$$

If $G$ and $\epsilon$ depend on $p$, we must write instead of (xv.)

$$
\frac{1}{8} \int_{0}^{\infty} \mathrm{C} \epsilon[f(p)]^{2} \cdot d_{l^{\prime}}, . . .+ \text {. (xvi.) }
$$

which then represents the amount of energy per volume CT stored up in the radiation given by (ii.). If we consider the effects of $\mathrm{Y}$ and $\mathrm{Z}$, we sball have to multiply expression (xvi.) by three, for homogeneous radiation.

$\S 5$. In the assumption represented by (iii.), we might have introduced terms involving waves moving parallel to $\mathrm{O} z$, in addition to those moving parallel to $\mathrm{O} y$. The final result is, however, the same, and we have not therefore considered these terms.

Another point to be noticed is this. The function $f(p)$ is a function of $\mathrm{T}$, the time-interval in forming (ii.). Thus $[f(p)]^{2} / \mathrm{T}$ must be a function of the temperature, as (xvi.) indicates, if there is a definite distribution of energy amongst the wave-lengths.

§6. It seems natural to ask what is the effect of introducing damping terms $\epsilon^{-\alpha p y / c}$ in (vii.), $\epsilon^{-\alpha q y / C}$ in (viii.), where $\alpha$ is a coefficient of absorption which is ultimately to be taken as vanishing. The integral which arises corresponding to $(x . a)$ is interesting from the point of view of the pure 
mathematician, and it would be interesting to have the couditions worked out on which such an integral would give as limit the result (xii.) above arrived at.

The question, however, is closely bound up with the expansion of an arbitrary function not in terms of sines and cosines as in the ordinary Fourier expansion, but in terms of what may be called exponentially damped sines and cosines.

$\S 7$. The results above obtained indicate that in the random impulses that constitute natural radiation, we may be led mathematically to a certain distribution of energy in the spectrum, provided that the time-interval $T$ contains $a$ sufficiently large number of any periods considered. Thus between the values $p$ and $p+d p$ we shall have an amount of energy $\epsilon[f(p)]^{2} d p / 8 \mathrm{~T}$ per unit volume. If we identify this with the steady distribution obtained by experiment, we are led to suppose that the harmonic analysis of a component of (say) the electric torce $\mathrm{X}$ over a sufficiently long time $\mathrm{T}$, must yield ultimately results of a steady type in such a way that $e . g$. if $\mathrm{X}$ be given by (ii.), $\left[f^{\prime}(p)\right]^{2}$ tends to become proportional to $\mathrm{T}$, the time over which the original disturbance is analysed. Of course the exact character of $f(p)$ cannot be settled by the considerations adduced.

LXXXVII. On the Mechanism of Molecular Action. (A Contribution to the Kinetic Theory of Gases.) By T. CARLTON Surron, B.Sc., Gorernment Research Scholar in the Lnirersity of Melbourne*.

\section{Introduction.}

THERE are a number of problems which may be attacked 1 by means of either thermodynamics or the kinetic theory; and the results obtained by each method should be in good agreement with experiment and with one another.

Up to the present, much more has been done by means of the thermodynamical than the kinetic method. 'This is due no doubt to the fact that, while the elements of thermodynamics are well known, there is as yet no knowledge of those mechanisms of ultimate molecular action on which the further development of the kinetic theory must be based.

Accepting the thermodynamical results, it should be possiole to find certain such mechanisms which will yield kinetic results in agreement with the thermodynamical.

If, in addition, these results are in good agreement with experiment, there is strong evidence in favour of the assumed mechanisms.

* Communicated by Professor Lyle, M.A., D.Sc., F.R.S. 